\title{
Fast Insolation Computation in Large Territories
}

\author{
Siham Tabik ${ }^{1}$, Jesús M. Vías ${ }^{2}$, Emilio L. Zapata ${ }^{1}$, and Luis F. Romero ${ }^{1}$ \\ ${ }^{1}$ Depto de Arquitectura de Computadores, campus de Teatinos, 29080 Málaga, Spain \\ 2 Depto de Geografía, campus de Teatinos, 29080 Málaga, Spain \\ siham@ac.uma.es
}

\begin{abstract}
This paper presents a new model for the computation of the insolation in large territories. The main novelty of this work consists in that it reduces significatively the number of necessary arithmetic operations, and simplifies the calculations introducing errors that are inferior to the ones imposed by the climate in a territory. In particular, a faster algorithm to compute the horizon at all points of a terrain is provided. This algorithm is also useful for shading and visibility applications. Moreover, parallel computing is introduced into this model using grid computing technology to extend its application to very large territories.
\end{abstract}

Keywords: Insolation, horizon computation, very large territories, Globus.

\section{Introduction}

The knowledge of the amount of incoming solar energy at diverse geographic locations is of a paramount importance in several fields, in solar energy utilization, agriculture, forestry, meteorology, environmental assessment and ecology. However, most solar radiation models that take into account the topographic heterogeneity and climatological features, like solar analyst [1] and r.sun model [2] have a large computational cost and high storage requirements, which become unapproachable for large territories. This paper presents a fast and accurate model that computes the insolation in large terrains considering all the involved factors, i.e., the localization and the horizons of all the points of the territory, the localization of the sun and the meteorological factors. We demonstrate that a great part of calculations can be reduced substantially by searching equivalences, until reducing it to the minimum possible. In addition, an implementation in grid computing have been developed to allow the extension of this model to very large territories, of the order of millions of kilometers. We describe the performance results of the application of the model to Andalucia territory.

\section{The Sky}

The sun passes from many parts of the sky. In each one of these localization, the incoming radiations into a horizontal surface have different orientation (or azimuth) $\alpha$ and inclination (or altitude) $\Theta$. If in addition, each point of the 
territory has different orientation head and inclination tilt, a priori, it would be necessary to know the incidence in each point of the territory, at each instant of the year.

However, the sun passes many times form the same zone of the sky. Our first simplification consists in supposing that the considered territory is horizontal and construct a celestial map of hemispherical shape that covers the horizon. We discretize this map into cells, and determine the quantity of radiation received by each point of the territory from each one of these cells. According to the spherical coordinates, the discretization results in $N_{s}=90 \times 360$ celestial cells that can be represented by a matrix of 90 lines and 360 columns. Each coefficient of this matrix correspond to a celestial cell with different coordinates $\alpha$ and $\Theta$.

The calculation of the position of the sun can be performed using the equations of the earth movement with respect to the sun [3].

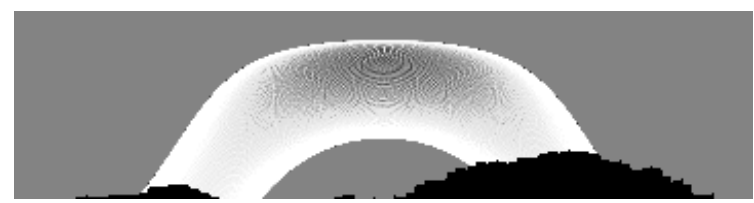

Fig. 1. Insolation of one year of exposition in a point of the horizon (black) displayed from the left to the right, in the west, south, east and north. The dark and light colors show low and high values of insolation respectively.

Performing these calculations, each cell of the obtained matrix of results accumulates the received radiation, i.e., the number of hours the sun has emitted energy from that cell. As illustration, Fig. 1 shows the insolation one year of exposition. The set of $N_{s}$ cells or celestial sectors are represented with the symbol $\Delta$.

The error produced in the discretization of the sky is lower than the committed by nature due to cloudiness. This error can be quantified in order to determine an appropriate dimension of celestial sectors.

\section{The Ground}

A terrain is most often represented with a digital elevation map (DEM), that can be discretized into cells. Typically, a very large territory contains millions of points, however, many of these $N$ points are similar. In particular, each localization $P$ has a given orientation head $_{i}$ and inclination tilt $_{i}$ with respect to the horizontal but with a very small error. We can consider that there exist only $N_{f}=90 \times 360$ possible orientations in our territory. Consequently, there exist only $N_{s}$ different cells, in which we want to compute the insolation. Let's consider $\Omega$ the set of those $N_{f}$ possible orientations. If there would be no mountains in the horizon, all points with the same orientation will receive the same quantity of radiation, that we call $E_{\text {full }}\left(\right.$ head $_{i}$, tilt $\left._{i}\right)$. 
For each one of the $N_{s}$ different cases we compute the insolation received from the corresponding $N_{s}$ celestial sectors $\Delta$, in which the angle of incidence is taken into account, this can be computed with only $5 \times N_{f}$ trigonometrical operations for each element $\varnothing$ of $\Omega$. In total, we obtain $5 \times N_{f} \times N_{s}$ operations.

Up to here, all the calculations are performed with a low computational cost.

\section{The Horizon}

Each point of the total $N$ points of a territory suffers from a partial concealment of the sun due to the orography that surrounds it (from the orto to the astronomical decline). If we consider that the orography is different in each point of the territory, the computation becomes very complicated. We will have $N \times N_{s}$ possible cases, where $N$ is a very large number, of the order of millions. In addition, an accurate computation of solar occultation requires an extraordinary amount of arithmetical operations as discussed in next section.

\subsection{The Calculation of Shaded Zones}

In principle, to determine the shadowed regions for each position of the sun we should first compute the trajectory of the sun rays for each terrestrial cell. However, the execution of such calculation, from this perspective, that we could call celestial, implies a large redundant calculation. Herein, we have chosen a different vision of the problem. In particular, we compute the visibility of the celestial cells, through which sometimes the sun has passed, from any cell of the territory. In other words, we are interested in knowing the profile or the horizon of a territory.

Tackling the problem from this perspective, that we could call terrestrial, is absolutely equivalent, besides, this has some advantages that we reveals in next sections.

Generally, the horizon of each point must analyzes the position of the rest of points of the grid (i.e., the territory), with a computational complexity $O(N)$. The computation of the horizon of all these points is $O\left(N^{2}\right)$, which implies an impractical computational cost, i.e., a N-body problem.

However, there exist several algorithms that simplify the calculations. For example the approximative method of Cabral, Max and Springermeyer (CMS) 4 divides the horizon in $s$ sectors, and in each sector it determines the elevation of the horizon considering solely the points of the terrain that are in the central line of the sector. This algorithm has a computational cost $O\left(s\left(N^{1.5}\right)\right)$. Stewart 5 proposed a more precise algorithm that computes the horizon in all the sector with a cost $O\left(s N\left(\log ^{2} N+s\right)\right)$. This method is faster than the CMS, and the quality of the image generated is better especially for very large terrains, i.e., more than 100.000 points.

\subsection{Stewart Model and the Horizon Computation}

Stewart [5] divides the horizon of each point $P$ in $s$ sectors $\sigma_{i, p}(1 \leq i \leq s)$ that form an angle $\frac{2 \pi}{s}$ radians each one. 
This algorithm consists in performing for each sector $i$, four important steps:

1. compute the new coordinates of all the points in the coordinate system $\left(a^{\perp}, b^{\perp}, z\right)$, where $a$ and $b$ are two horizontal vectors in the directions $\frac{2 \pi}{s} i$ and $\frac{2 \pi}{s}(i+1)$ respectively, and sort all the points by $a^{\perp}$ and $b^{\perp}$ for each direction

2. compute the horizons using the last updated hull structure

3. update the hull structure including the information of the considered point

4. insert the horizon of the considered point in the original structure

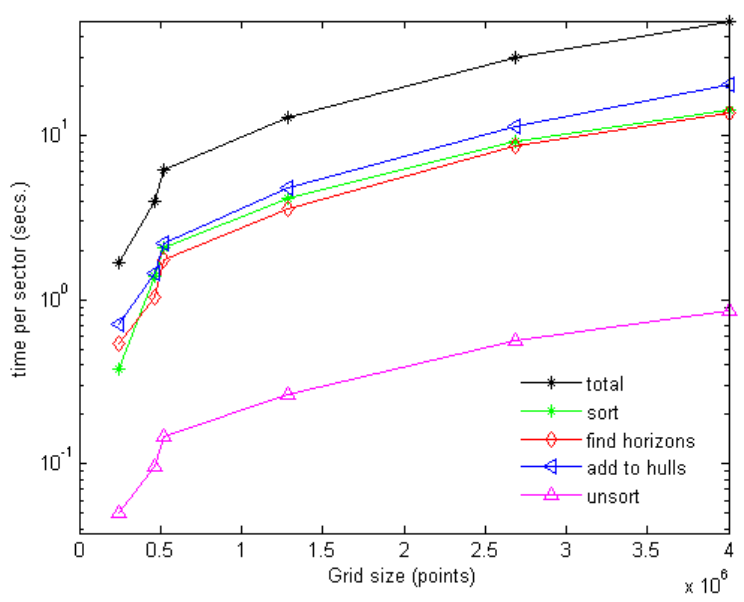

Fig. 2. Times by sector in logarithmic scale of the four main steps of Stewart's algorithm

Figure 2 shows the time per sector of each step of Stewart's algorithm, for several grid sizes. As it can be seen from this figure, the time increases enormously as the size of the grid increases. This means that the calculation of the horizon of Andalucia DEM that has $100000 \times 50000$ cells of precision $10 \times 10 \mathrm{~m}^{2}$ will suppose very long execution time. However, dividing this DEM into subgrids of $2000 \times 2000$ cells requires only $256 \mathrm{MB}$, which is reasonable from a computational point of view but this produces an undesired edge effect as explained in next section.

\subsection{Our Horizon Algorithm}

In this work we propose an optimized algorithm that in addition to compute the horizon of large terrains in a reasonable time, it eliminates the edge effect due to the division into regular grids. First, to eliminate the edge effect we apply a two-partition technique that consists in considering the horizon of a given point as the overlapping of its horizons in the grid $\mathrm{A}$ and $\mathrm{B}$, both of size $10 \times 10$ points. Each point $\mathrm{P}$ belongs to a grid in the division A (black grid in Fig. 3) 


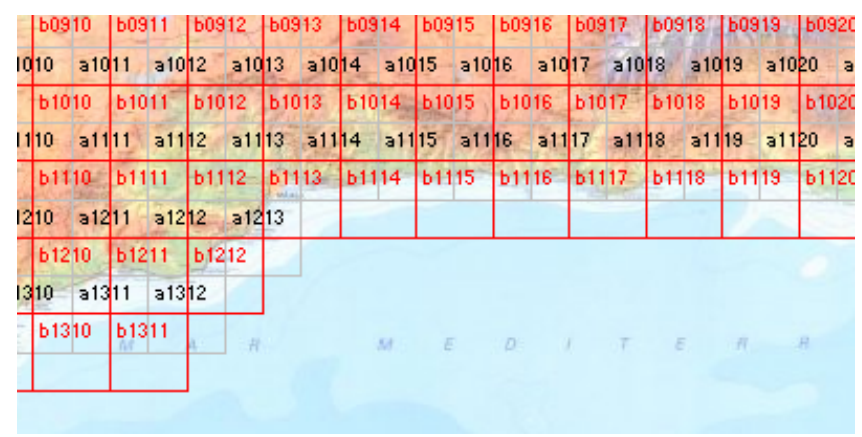

Fig. 3. Two-partition technique. Grid A (black color) and grid B (grey color).

and an other grid in the division B (grey grid in Fig. 3). Each single grid $A_{i, j}$ is considered as four sub-grids: $A_{i, j}^{n w}, A_{i, j}^{n e}, A_{i, j}^{s e}$ and $A_{i, j}^{s w}$, where each of which belongs to four B-grids (see Fig. 3 ) in such a way that:

$$
A_{i, j}^{n w}=B_{i, j}^{s e}, A_{i, j}^{n e}=B_{i, j+1}^{s w}, A_{i, j}^{s e}=B_{i+1, j+2}^{n w} \text { and } A_{i, j}^{s w}=B_{i+1, j}^{n e}
$$

The horizons are computed only in the opposite direction of the position of each one of the four sub-grids inside the grid, for example, a sub-grid $n w$ computes its horizon only in the direction se. This reduces the time of step 2 in the algorithm by half.

To compute the horizons of more than $10 \mathrm{~km}$, we have used a low precision grid that we call $\mathrm{C}$ grid, of dimension $2000 \times 1000$ points, which covers Andalucia with steps of 200 meters.

As stated before, Stewart's algorithm computes the horizon in a main loop by sector. The time taken in computing the horizons of Andalucia using Stewart's algorithm is about ( 518 grids $\times 64$ sectors $\times 50$ seconds) equivalent to 19 days. However, taking into account the regularity of the grid and applying some optimizations, this time can be significantly reduced.

- Our first optimization consists in reusing the sorting of sector $i$ by $b^{\perp}$ for sorting sector $i+1$ by $a^{\perp}$. Thus, we reduce the computational time of step 1 to approximately half the time. This optimization can be applied to all sectors except the first one.

- The second optimization consists is that in a regular grid, there exist an easier way to sort the points by $a^{\perp}$ and $b^{\perp}$ without the need to compute its coordinates in that coordinate reference. It can be done by just counting the number of points behind the running line, using very simple algebraic operations. As the position number is directly computed, it is not necessary to use any sorting algorithm. In practise, the time spent in the optimized phase 1 is NULL.

- Our third optimization is the use of the two-partition technique presented here, so that we do not need to perform the 2 nd phase for every sector, but only for the half of them, as the horizon for the other half is obtained from the overlapped grid B (and vice versa). 
The calculated horizon by the optimized algorithm is stored in a matrix $\mu$ of dimension $1 \times 360$.

As a result of the proposed optimizations, the expected computational time is reduced to less than 11 days. The improvement in runtime when applying the optimizations 1,2,3 and 4 is evident as shown in Fig. 4.

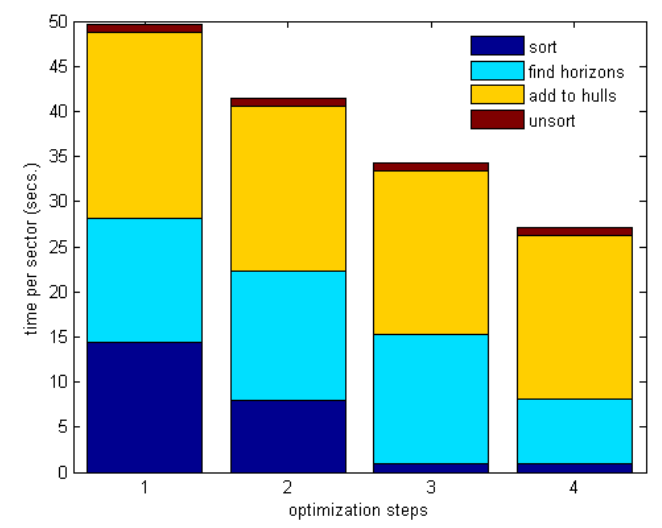

Fig. 4. The improvement in times applying the optimizations $1,2,3$ and 4 for a grid of size $2000 \times 2000$

\subsection{Parallel Computing}

An important quality of the proposed horizon algorithm is that it does not include any data dependencies between sectors, which means that parallelism can be easily applied. By using dual core technology, the execution time for any grid is reduced by half (about 18 second per grid), and just a week for the whole territory.

Although it was unnecessary to exploit more parallelism in Andalucia project, we have employed the Grid technology and Globus [6], to reduce even more the computational time, and to extend the possibilities to tackle very large territories. With this technology, and using the computational power of a laboratory with 32 dual-core processors, the horizons for Andalucia has been computed in only 5 hours. Where the grids A and B are of dimensions 268 and 250 respectively.

\section{The Accumulated Sky}

As mentioned in section 3 , each element $\varnothing$ of the set $\Omega$ watches the sky from the same perspective, but with different horizons in each case. This means that each element $\varnothing$ of $\Omega$ (or a point $P$ of the territory) have a horizon $\mu_{i}$, with $\mu_{i} \in \Lambda$. Being $N$ the total number of elements of $\Lambda$; as there are so many horizons as points in the territory we obtain $N \times 32400$ operations.

But, for each element $\varnothing($ head,tilt $)$ of $\Omega$, there exist only 90 possible heights for the obstacles that can have in each azimuth $\alpha$. The received insolation by 
a given point $P$ from the celestial cells column $k$ of azimuth $\alpha(k)$ could be calculated adding the energies received by all the celestial cells of that column from the zenith $\left(90^{\circ}\right)$ to the correspondent obstacle $\mu_{i}(k)$. Let's define $E_{\text {real }}\left(\alpha_{i}, \theta_{i}, k, \mu_{i}(k)\right)$ as the insolation in the element $\varnothing$. The resulting matrix is of dimension $32400 \times 32400$, and stores the values of $E_{\text {real }}$.

In order to compute the yearly insolation matrix, we have to redefine our representation of the celestial map, modifying it in such a way that each celestial cell stores the received energy in the whole year, adding the values of the column that goes from the zenith to that cell.

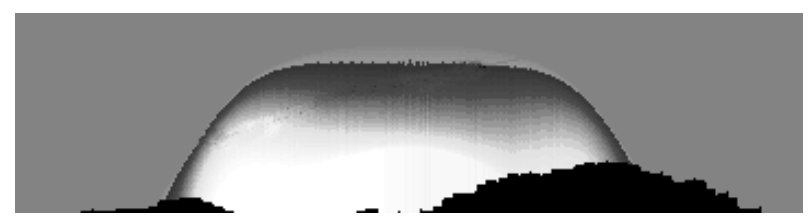

Fig. 5. The insolation of one year computed using the accumulative representation of the celestial matrix

Let's $\sum \Delta$ be the new accumulative celestial matrix, of dimension $32400 \times$ 32400. Our definitive model for the computation of one year insolation in large territories can be resumed as follows:

- For each minutes of the year $(365 \times 24 \times 60$ minutes $)$

- Compute the position of the sun. The resulting celestial matrix is of dimension $90 \times 360$ )

- For each possible orientation in the territory

- Compute $E_{\text {full }}$ received from all $N_{s}$ celestial cells in absence of horizons

- Compute the matrix $E_{\text {real }}$ of one year accumulated insolation in each celestial cell

- For each point $\mathrm{P}$ of the territory

- Determine its coordinates $(i, j)$

- Compute its horizon $\mu_{i}$ (a matrix of dimension $1 \times 360$ points $)$

- For each column $k$ of the celestial matrix

* Compute the accumulate insolation taking into account its corresponding horizon $\mu_{i, k}$

If the terrestrial cell is horizontal:

$$
E(i)=E_{\text {full }}\left(a z m_{i}, e l v_{i}\right)
$$

If the terrestrial cell is a mountainous site:

$$
E(i)=\sum_{a z m_{k}=0}^{360} E_{\text {real }}\left(\text { head, tilt }, \text { azm } k, \mu_{i, k}\right)
$$


For the analysis of the attenuation of solar radiation passing through clouds, we have extrapolated the behavior of cloudiness in the past to the future. This hypothesis is true if we neglige the effect of global change.

The calculation of the insolation taking into account the cloudiness for a grid of dimension $2000 \times 2000$ using our model takes 25 seconds.

\section{Conclusions}

From a computational point of view, this paper has proposed an efficient model to compute the insolation in large and very large territories. In particular, an algorithm for horizon computation about two times faster that the fastest algorithm provided in literature has been provided. In addition, a parallel implementation of the horizon algorithm in grid computing has been carried out to make possible the study of very large territories, which is useful for different applications, including shading and visibility ones.

\section{Acknowledgment}

This work was supported by the Spanish Ministry of Education and Science through grants TIC2003-06623.

\section{References}

1. Fu, P., and Rich, P.M.: The solar analyst 1.0 User Manual. WWW document, http://www.fs.fed.us/informs/solaranalyst/solar_analyst_users_guide.pdf, 2000.

2. Marcel, S., Jaroslav, H.: A New GIS-based Solar Radiation Model and Its Application to Photovoltaic Assessments. Transaction in GIS, 8 (2004) 175-190.

3. Page, J., K.: Prediction of Solar Radiation on inclined surfaces. (1899) Springer.

4. Cabral, B., Max, N., Springmeyer, R.: Bidirectional reflection functions from surface bump maps. Computer Graphics, Proceedings of SIGGRAPH'87, 21 (1987) 273-281.

5. James, A., Stewart.: Fast Horizon Computation at all Points of a Terrain with Visibility and Shading Applications. IEEE Trans. on Visualization and Computer Graphics, 4 (1998) 82-93.

6. http://www.globus.org/toolkit/ 